Case Report

\title{
A rare presentation of enterocutaneous fistula
}

\section{Devi Thirunavukarasu*, Sriram Khanna, Shanthi Ponnandai Swaminathan, R. Kannan}

Institute of General Surgery, Madras Medical College and Rajiv Gandhi Government General Hospital, Chennai, Tamil Nadu, India

Received: 07 January 2022

Accepted: 02 February 2022

*Correspondence:

Dr. Devi Thirunavukarasu,

E-mail: devit15aug@gmail.com

Copyright: $\odot$ the author(s), publisher and licensee Medip Academy. This is an open-access article distributed under the terms of the Creative Commons Attribution Non-Commercial License, which permits unrestricted non-commercial use, distribution, and reproduction in any medium, provided the original work is properly cited.

\section{ABSTRACT}

Enterocutaneous fistula is an abnormal communication between two epithelialized surfaces, one of which is a hollow viscous organ. It most commonly occurs as a surgical complication. Other causes are trauma, malignancy, inflammatory bowel disease, ischemia. The great majority of enterocutaneous fistula are iatrogenic $(75-85 \%)$ and rest $(15-25 \%)$ occur spontaneously. Enterocutaneous fistula is the one of the most challenging conditions managed by General surgeon. The mortality rates vary in different series for patients, with enterocutaneous fistula and remains $5 \%$ to $15 \%$. Here we report a rare presentation of enterocutaneous fistula.

Keywords: Enterocutaneous fistula, Previous surgery, Abscess drainage, Nutritional support, Definitive surgery

\section{INTRODUCTION}

Enterocutaneous fistula is one of the most challenging conditions managed by general surgeon. Regarding morbidity and mortality of an enterocutaneous fistula, Edmunds et al. describes a classic triad as sepsis, malnutrition and fluid and electrolyte imbalance. ${ }^{1}$ The mortality vary in different series for patients with enterocutaneous fistulas and remains $5 \%$ to $15 \% .^{2-5}$ Spontaneous fistula complicating cancer usually occur in advanced disease and the prognosis is poor.

\section{CASE REPORT}

A 65-year female came to our emergency department with the history of swelling in the right lumbar region for 7 days, approximately $5 \times 3 \mathrm{~cm}$ swelling with ulceration in the right lumbar region. There was no warmth and tenderness. Fluctuation present. High frequency ultra sound shows $4 \times 3 \mathrm{~cm}$ hypoechoic collection noted as subcutaneous abscess. Patient was prepared for surgery. Incision and drainage was done. On pod 2, there was a solid fecal matter drained through the surgical site. Then patient was re-evaluated and x-ray abdomen erect and lateral shows some metal particles. Then the patient gave history of surgery when she was 10 years old and complete details not known. Contrast CT abdomen showed extravasation of contrast through the fistulous tract in ascending colon. Then patient diagnosed as enterocutaneous fistula. Patient was on stoma bag with nutritional supports, electrolyte correction and discharged and reviewed. On follow-up after 3 months patient was taken electively and limited resection of ascending colon fistula, Colo-colic anastomosis with latissimus Doris flap.

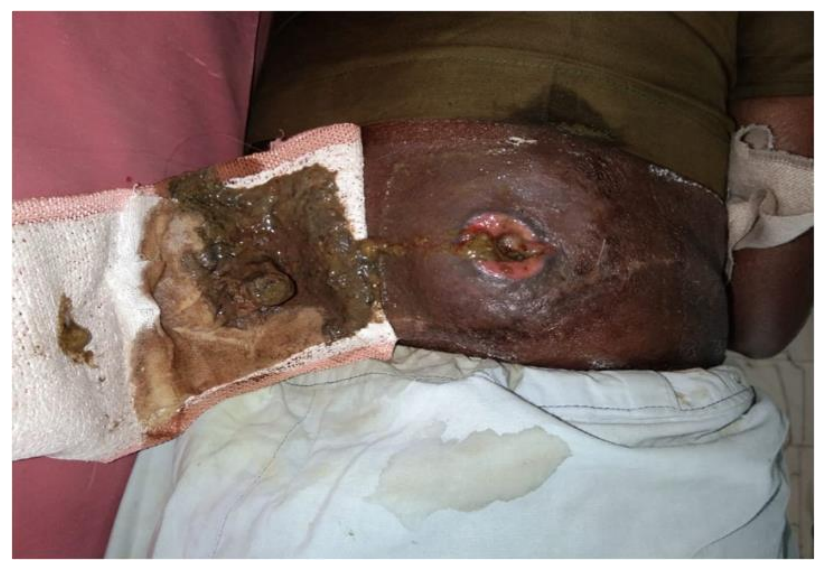

Figure 1: Fecal discharge from incision site on pod 2. 


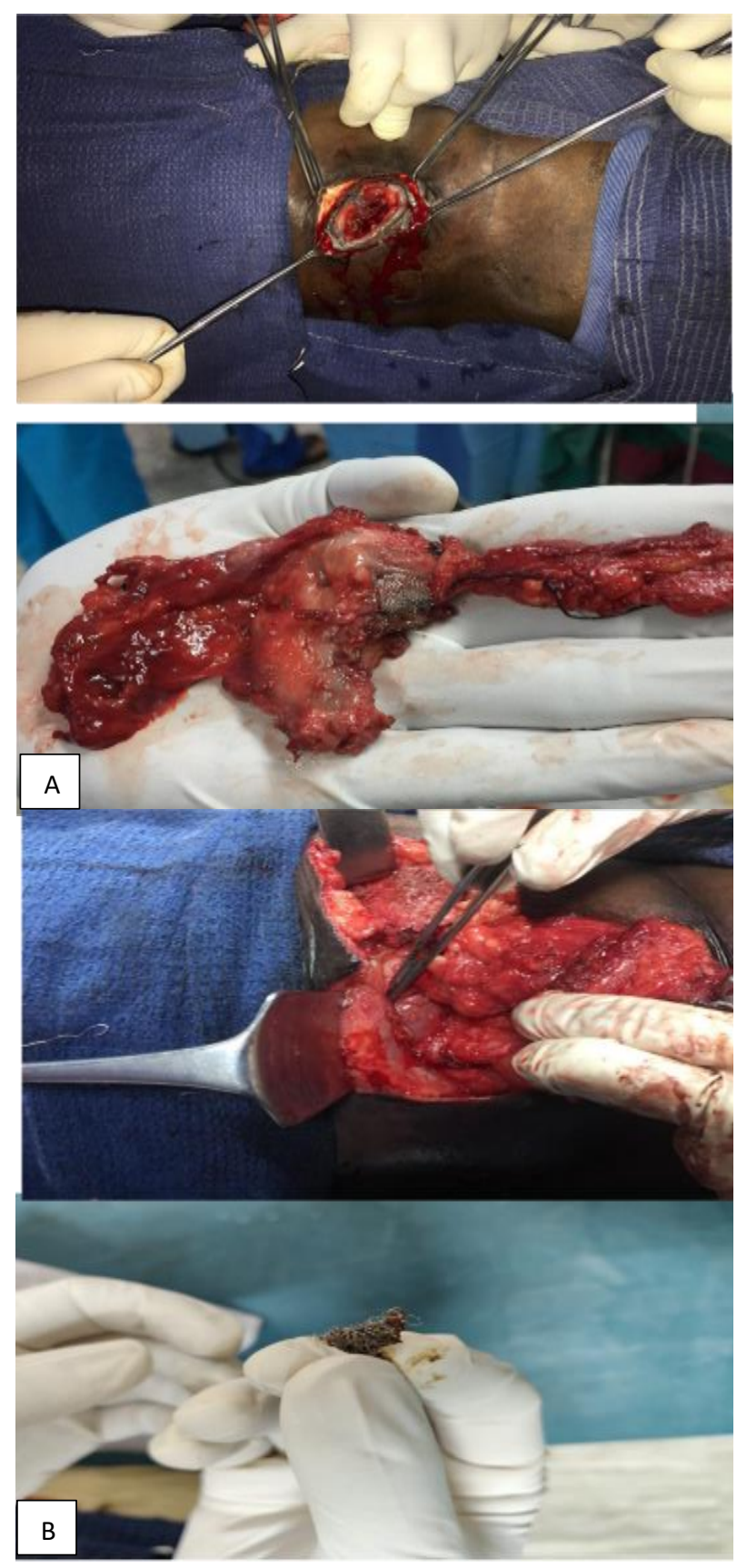

Figure 2 (A and B): Limited resection of ascending colon fistula and colo-colic anastomosis with latissimus dorsi flap.

\section{DISCUSSION}

Enterocutaneous fistula is an abnormal connection between the intra-abdominal gastrointestinal tract and skin or wound. Enterocutaneous fistula is one of the most challenging conditions managed by general surgeon. Regarding morbidity and mortality of an Enterocutaneous fistula, Edmunds et al describes a classic triad as sepsis, malnutrition and fluid and electrolyte imbalance. ${ }^{1}$ The mortality vary in different series for patients with enterocutaneous fistulas and remains $5 \%$ to $15 \%$. $^{2-5}$ Spontaneous fistula complicating cancer usually occur in advanced disease and the prognosis is poor. Enterocutaneous fistula can present in different ways. Any abdominal swelling or mass should be evaluated carefully with detailed history, clinical examination and Imaging studies. With adequate nutritional support, electrolytes correction and control of sepsis, definitive surgery can be done.

Enterocutaneous fistula result from one of the following several conditions: 1. Extension of bowel disease to surrounding structures, 2. Extension of adjacent non bowel disease to normal bowel, 3. Inadvertent or unrecognized injury to the bowel and 4. Anastomosis disruption.

Enterocutaneous fistula can be classified in many ways based on output, etiology and source. Iatrogenic fistula constitutes about $75 \%$ to $85 \%$ and rest $15 \%$ to $25 \%$ are spontaneous. Common causes for spontaneous fistula are inflammatory bowel disease (most common), diverticular disease, malignancy, radiation, tuberculosis, actinomycosis, appendicitis and ischemic bowel perforation. The conservative approach's closure rates are about 20 to $30 \%$. $^{6-7}$ About $75 \%$ to $85 \%$ of the fistula is iatrogenic and occurs due to surgical procedures and trauma complications. Patient may present with abdominal pain, distension, tenderness, fever, discharge of enteric contents from a drain, or main abdominal wound. The patient may present with complications including sepsis, fluid and electrolyte derangements and malnutrition. About $70 \%$ of patients with enterocutaneous fistula have malnutrition due to loss of protein-rich enteric contents, intra-abdominal sepsis, and electrolyte imbalance. Investigations include complete hemogram and serum electrolytes, serum transferrin level $<200$ $\mathrm{mg} / \mathrm{dl}$ is a predictor of poor healing, elevated serum albumin and CRP indicates infection. ${ }^{8}$ Imaging includes fistulography, contrast enhanced CT abdomen done to know the origin of fistula, length of fistulous tract. The goal of treating enterocutaneous fistula is to restore bowel continuity, to achieve enteral nutrition and to close the fistula. Initial resuscitation with intravenous fluids and correction of electrolytes. Control sepsis with adequate drainage and antibiotics. The best nutritional support is a combination of parenteral and enteral support. Somatostatin and its long-term analog octreotide have also been proposed in the management of the enterocutaneous fistula to decrease output. However, it is not clear that octreotide has any beneficial long-term effect on spontaneous closure except in biliary and pancreatic fistulas. Skin and wound care management includes simple gauze dressing, negative pressure wound therapy, vacuumed Assisted closure. All patients with enterocutaneous fistula initially put on conservative management for the first 4-6 weeks. The factors which favours spontaneous closure are surgical etiology, diverticulitis, transferring $>200 \mathrm{mg} / \mathrm{dl}$, no obstruction, no infection, low output fistula, no sepsis, balanced electrolytes. ${ }^{8,9}$ Patient with no associated adverse factors should wait for about 3-6 months before planning surgery 
for an enterocutaneous fistula. High volume ECF care centers are currently reoperating on patients at 6 to 12 months. Waiting longer than 12 months in those patients who have large wounds and potential hernia may cause loss of domain and complicate hernia repair. Psoas muscle density is a predictor for poor healing, which can reflect sarcopenia. ${ }^{10}$

\section{CONCLUSION}

The treatment of enterocutaneous fistula one of the most challenging problems in surgery. Despite recent development in intensive care and nutrition support, mortality rates are very high. A multidisciplinary approach is required for the proper management of enterocutaneous fistula. The primary aim is a restoration of gastrointestinal continuity with limited morbidity and mortality and fistula recurrence.

Funding: No funding sources

Conflict of interest: None declared

Ethical approval: Not required

\section{REFERENCES}

1. Edmunds L, Williams GM, Welch CE. External fistulas arising from the gastrointestinal tract. Ann Surg. 1960;152:445-71.

2. Joyce M, Dietz W. Management of complex gastrointestinal fistula. Curr Probl Surg. 2009;46(5):384-430.

3. Owen R, Love TP, Perez SD, Srinivasan JK, Sharma J, Pollock JD et al. Definitive surgical treatment of enterocutaneous fistula. JAMA. 2013;48(2):118-26.
4. Lynch AC, Delaney CP, Senagore AJ, Connor JT. Clinical outcome and factors predictive of recurrence after enterocutaneous fistula surgery. Ann Surg. 2004;240(5):825-31.

5. Ruben G. Treatment strategies in 135 consecutive patients with enterocutaneous fistulas. World J Surg. 2008;32:445-53.

6. Rahbour G, Gabe SM, Ullah MR. Seven-year experience of enterocutaneous fistula with univariate and multivariate analysis of factors associated with healing: development of a validated scoring system. Colorectal Dis. 2013;15(9):1162-70.

7. Haffejee AA. Surgical management of high output enterocutaneous fistula: a 24-year experience. Curr Opin Clin Nutr Metab Care. 2004;7(3):309-16.

8. Lloyd DA, Gabe SM, Windsor AC. Nutrition and management of enterocutaneous fistula. Br J Surg. 2006;93(9):1045-55,

9. Martinez JL, Luque-de-Leon E, Mier J, BlancoBenav des R, Robledo F. Systematic management of postoperative enterocutaneous fistula;factors related to outcome World J Surg. 2003;323-44.

10. Lo WD, Evans DC, Yoo T. Computed Tomography measured Psoas density predicts outcomes after enterocutaneous fistula Repair. JPEN J Parenter Enteral Nutr. 2018;42(1);176-85.

Cite this article as: Thirunavukarasu D, Khanna S, Swaminathan SP, Kannan R. A rare presentation of enterocutaneous fistula. Int Surg J 2022;9:753-5. 